\title{
Bringing Digital Storytelling to the Mobile
}

\author{
Thomas Reitmaier and Gary Marsden \\ University of Cape Town Centre in ICT4D \\ Cape Town, South Africa \\ Thomas.Reitmaier@uct.ac.za, gaz@cs.uct.ac.za
}

\begin{abstract}
Technology has changed the way in which people tell their stories. This paper introduces digital storytelling and looks at why the mobile is an ideal platform for creating digital stories. The iterative design approach chosen for our Mobile Digital Storytelling system is discussed. Results of a final experiment, comparing our system to an existing mobile system that supports digital storytelling, are presented, which suggest that our system has met its design goals of providing an effective and efficient user interface. Qualitative insights from user evaluations show that mobile digital storytelling has a future.
\end{abstract}

Keywords: User-centered design, digital storytelling, mobile devices, content creation, interaction design.

\section{Introduction}

Everybody has stories to tell. They can range from the banal, to the instructional, and on to rich and compelling stories. Although the content of these stories varies considerably, one aspect holds true for all of them - they are all stories and thus convey an experience.

Storytelling serves as "dialog between people, cultures, and times" [4]. Originally an oral tradition, it has evolved into a written practice, and has recently experienced a shift to the digital medium. Storytelling empowers people to "preserve, share, and reflect on life experiences" [4]. It has evolved from a strong oral background to utilizing new technological advances and media types as they have emerged [2].

\subsection{Digital Storytelling}

A broad definition of a digital story is that it is a series of pictures with a voice-over or describing text. A clear definition of a 'digital story', however, does not exist. Some researchers say that digital storytelling should involve a degree of critical reflection [4]. To them a digital story is much more than an annotated photo slideshow. The story, not the pictures, is the most important aspect. Others argue that the term 'digital story' is more of an umbrella, and encompasses both 'picture-driven' and 'story-driven' approaches [1].

\subsection{Mobile Digital Storytelling}

Bringing digital storytelling to the mobile, while challenging, offers great opportunities. It would allow users without access to a personal computer to create and share 
their stories, thus giving them a digital voice. Digital stories are often personal stories, in tune with the personal nature of the mobile and the emotional attachment users have towards it [3]. This compatibility suggests that the mobile is an ideal and low cost digital storytelling platform.

Some of the challenges we faced while developing our mobile digital storytelling system are the limited computing resources of mobile phones and their limited interaction capabilities. By restricting ourselves to simpler digital stories, consisting of still images annotating a recorded audio narrative, our mobile digital storytelling system should run on lower end mobiles. We chose an iterative design approach, outlined in the next section, to tackle the limited interaction capabilities of mobiles.

\section{Design Process}

The design question was to develop an easy-to-use mobile system, which allows people to record their stories. Everybody has a story-not just people with access to computers - by bringing digital storytelling to the mobile we aim to extend its reach.

One of the few existing systems that supports mobile digital storytelling is the Mobile Multimedia Presentation Editor [2] by Jokela et al, seen in the left of Figure 1. After inspecting the Mobile Multimedia Presentation Editor, we decided to steer clear of their timeline interface and rather try to improve upon it. Timeline interfaces may be familiar to many of us from video-editing software, but they may be difficult to use, particularly in the storytelling context, for novice or casual computer users.
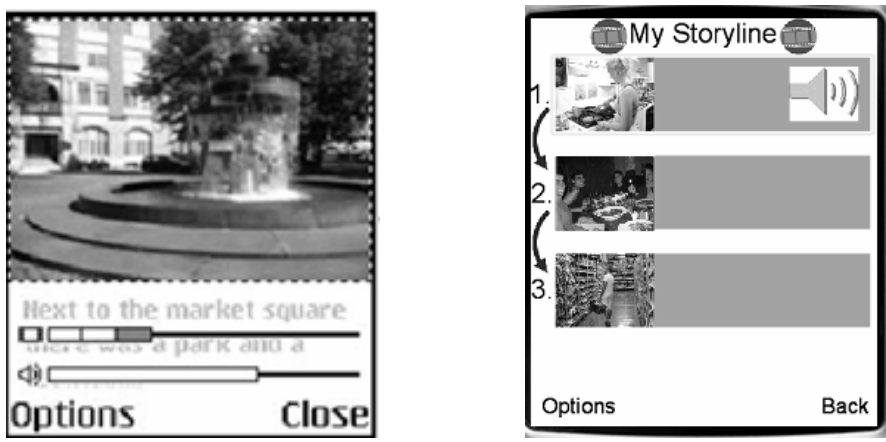

Fig. 1. The timeline interface of Mobile Multimedia Presentation Editor (left) and our Mobile Digital Storytelling storyline interface (right)

An approach based on the principles of Interaction Design was used to develop our Mobile Digital Storytelling system. In developing our system, we focused on three main activities, understanding user needs, developing prototypes, and user evaluation. Through iterative prototyping and user evaluation a final design emerged.

We created a prototype, which we improved and refined over four design iterations. In our background research, we established that two approaches could be taken towards creating a digital story, a story-driven (annotating narrative with pictures) and a picture-driven (adding narrative to pictures) approach. We decided to create two 
interfaces - one for each approach. User evaluations revealed that users preferred the picture-driven approach, which we will discuss from here on out.

In the first design iteration, we created a low-fidelity paper prototype, which we tested against usage scenarios and evaluated heuristically. Because of the interactive nature of our system we chose a user-less evaluation for this first design iteration.

We then incorporated the lessons learned from the first design iteration into the PowerPoint prototype of the second design iteration. We evaluated the usability of this prototype using six users. Because of time and cost constraints, the users we recruited for this evaluation were university students. To ensure sample population diversity, we recruited students from different parts of the university campus.

Keeping the lessons learned from previous design iterations in mind, we created a high-fidelity Flash Lite prototype, which runs on a mobile phone. In this prototype the system's interaction capabilities are more accurately modeled than in lower fidelity prototypes, giving an accurate model of the systems look, feel, and interactions. Again, we performed a formative user evaluation on the interface. As the interface was now more mature than the interfaces of previous iteration, we aimed to discover more subtle usability problems and thus decided to evaluate our system on eight student users, recruited from different university departments to ensure population diversity. The user evaluation performed on the Flash Lite prototype revealed mostly minor terminology related usability problems, which we addressed in the final design.

\subsection{Final Design}

The elements of the final interactive Flash Lite prototype can be seen in Figure 2. Pictures are added to the storyline, for which an annotating narrative is then recorded.
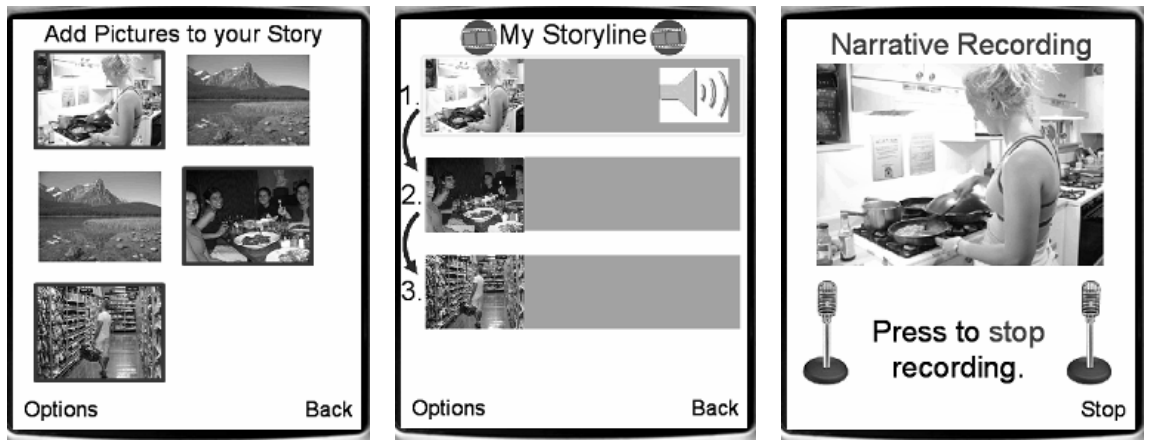

Fig. 2. Elements of the final interface design

In the previous iteration, mostly minor terminology related usability problems were discovered, which we addressed in this iteration. That led us to believe that an effective and easy to use design had emerged in our final prototype.

\subsection{User Experiment}

A summative evaluation was conducted in a usability lab to test the hypothesis that, given a pre-scripted story and annotating picture, users will be able to create a digital 
story more quickly using our Mobile Digital Storytelling system than using Mobile Multimedia Presentation Editor of Jokela et al.

The experimental results of the within-group experiment with counterbalancing testing the null-hypothesis show that all ten participants were able to perform the task of creating a digital story faster on our system (345 seconds on average) than on the Mobile Multimedia Presentation Editor (427 seconds on average). On average, the participants performed 82 seconds faster on our system-this constitutes a $23.9 \%$ improvement. A paired t-test performed $(\mathrm{t}=10.043)$ on the results showed a calculated p-value of less than 0.0001 , which is statistically significant. The experiment indicates that, when creating digital stories, users perform better (faster) on our Mobile Digital Storytelling system than on the Mobile Multimedia Presentation Editor of Jokela et al.

\section{Discussion and Conclusion}

We were surprised that very few users in our evaluations had ever heard of digital storytelling. After discovering digital storytelling, in part through interacting with our prototypes, our users were delighted with the simplicity, flexibility, and power of the digital story. Almost all of the users could immediately come up with a scenario where they could use our system, which ranged from "telling a friend about the club I'm currently at" to "I'm sure that the people in the AIDS clinic I volunteer at would love it"- showing the potential of digital storytelling and our mobile system.

We have seen not only that mobile digital storytelling is viable and possible but also that our system is a significant improvement to an existing system-enabling even storytelling novices to create and explore the power of digital stories.

Possible future work includes, more ethnographic work on how people tell stories using pictures, exploring locative aspects of storytelling, enabling users to collaborate on their stories, and better utilizing the ad-hoc nature of the mobile for in situ storytelling.

\section{References}

1. Balabanovic, M., Chu, L.L., Wolff, G.J.: Storytelling with digital photographs, 564-571 (2000)

2. Jokela, T., Lehikoinen, J.T., Korhonen, H.: Mobile multimedia presentation editor: enabling creation of audio-visual stories on mobile devices, 63-72 (2008)

3. Jones, M., Marsden, G.: Mobile Interaction Design. John Wiley \& Sons, Chichester (2006)

4. Landry, B.M., Guzdial, M.: Learning from Human Support: Informing the Design of Personal Digital Story-Authoring Tools. Journal of the International Digital Media and Arts Association 3(1), 106 (2006) 